\title{
Frontières
}

\section{Ériger des cimetières, construire l’identité}

Pratiques funéraires et discours nationaliste chez les Tigres

tamouls du Sri Lanka

\section{Cristiana Natali}

Volume 18, numéro 2, printemps 2006

La mort dans tous ses états

URI : https://id.erudit.org/iderudit/1073215ar

DOI : https://doi.org/10.7202/1073215ar

Aller au sommaire du numéro

Éditeur(s)

Université du Québec à Montréal

ISSN

1180-3479 (imprimé)

1916-0976 (numérique)

Découvrir la revue

Citer cet article

Natali, C. (2006). Ériger des cimetières, construire l'identité : pratiques funéraires et discours nationaliste chez les Tigres tamouls du Sri Lanka. Frontières, 18(2), 15-20. https://doi.org/10.7202/1073215ar

\section{Résumé de l'article}

Au début des années 1990, dans un Sri Lanka ravagé par la guerre civile, les Tigres de la Libération du Tamil Eelam (TLTE) ont modifié de manière significative les pratiques funéraires réservées à leurs combattants. À partir de cette période, les cadavres des combattants Tigres, jusque-là incinérés selon la tradition hindoue, ont commencé à être confiés à la terre. Ce changement a mené à l'établissement de cimetières Tigres (Tuillum Illam, littéralement " maison du sommeil »). Cet article traite des raisons qui ont fait des Tuillum Illam des lieux privilégiés pour le développement de la nouvelle identité nationale tamoule. 


\section{Résumé}

Au début des années 1990, dans un Sri Lanka ravagé par la guerre civile, les Tigres de la Libération du Tamil Eelam (TLTE) ont modifié de manière significative les pratiques funéraires réservées à leurs combattants. À partir de cette période, les cadavres des combattants Tigres, jusque-là incinérés selon la tradition hindoue, ont commencé à être confiés à la terre. Ce changement a mené à l'établissement de cimetières Tigres (Tuillum Illam, littéralement «maison du sommeil»). Cet article traite des raisons qui ont fait des Tuillum Illam des lieux privilégiés pour le développement de la nouvelle identité nationale tamoule.

Mots clés: cimetières - guerre Sri Lanka - Tigres tamouls - pratiques funéraires.

\begin{abstract}
At the beginning of the 90 s, in a Sri Lanka ravaged by civil war, the Liberation Tigers of Tamil Eelam (LTTE) decided to significantly modify funerary practices reserved to their fighters. Starting from that period the bodies of the Tigers, which until then were cremated in accordance with Hindu tradition, started being buried and thus entrusted to the land. This change has led to the institution of Tigers' cemeteries (Tuillum Illam literally "sleeping houses") which have become places of remembrance. This paper will elaborate on the reasons which have led to the identification of Tuillum Illam with privileged places for the development of the new Tamil national identity.
\end{abstract}

Keywords: cemeteries - war Sri Lanka - Tamil Tigers - funerary practices.

\section{ÉRIGER DES CIMETIËRES, CONSTRUIRE L'IDENTITÉ Pratiques funéraires et discours nationaliste chez les Tigres tamouls du Sri Lanka1}

\author{
Cristiana Natali, Ph.D., \\ anthropologue, chercheure, \\ Département d'études linguistiques et orientales, \\ Université de Bologne.
}

Cet article traite de la formation de l'identité nationale tamoule dans les zones nord-est du Sri Lanka, sous contrôle des Tigres de la Libération du Tamil Eelam (TLTE). Essentiellement, il sera question de l'importance des nouvelles pratiques d'inhumation dans le processus de construction d'une nation. Dans ce contexte, nous porterons une attention particulière à la perception que semblent avoir les Tamouls des cimetières Tigres, ces derniers étant perçus comme les centres symboliques de cette nouvelle entité politique qu'est le Tamil Eelam².

Dans notre étude, nous analyserons les raisons qui motivent cette perception. D'une part, nous proposerons des analogies fonctionnelles entre les cimetières des TLTE et les cimetières de guerre appartenant à la tradition militaire occidentale. D'autre part, nous mettrons l'accent sur cette par- ticularité qu'ont les cimetières des TLTE d'être perçus comme des lieux sacrés. Les cimetières des Tigres sont appelés Tuillum Illam, c'est-à-dire «maisons du sommeil », et ils sont souvent considérés comme des temples. Nous verrons que les TLTE, en dépit de leur nature séculière, ont décidé de ne pas rejeter cette interprétation religieuse, car elle leur permet d'inclure le Tuillum Illam dans le courant principal de la tradition hindoue. Cette habilité à s'intégrer à la sphère de l'hindouisme représente un élément crucial du processus de construction de la nation. Notre essai est issu d'observations lors d'un travail de terrain mené dans les régions du Nord-Est du Sri Lanka contrôlées par les TLTE, entre juillet 2002 et janvier 2003.

\section{LE CHANGEMENT DES PRATIQUES FUNÉRAIRES}

Au début des années 1990, dans un Sri Lanka ravagé par la guerre civile, un grand changement est survenu dans les rituels funéraires réservés aux combattants des TLTE. C'est à partir de cette date que 
les TLTE ont commencé à inhumer leurs morts et à les réunir dans les Tuillum Illam. Avant cette période, les corps des Maaveerar ("grands héros») étaient incinérés, en accord avec la tradition hindoue, et leurs cendres étaient confiées à leur famille.

Pour comprendre la signification de ce changement dans le rituel, nous devons d'abord comprendre quelles étaient les pratiques funéraires mises en place dans le Nord et dans l'Est du Sri Lanka. Ces pratiques dépendent de la foi professée par la famille du défunt. Si les chrétiens et les musulmans enterrent leurs morts, plaçant les corps dans leurs cimetières respectifs ${ }^{3}$, les hindouistes les incinèrent, dispersant ensuite les cendres dans les fleuves (nous parlerons des exceptions par la suite). En ce sens, l'inhumation n'est pas socialement inconnue mais réservée aux seuls chrétiens ou musulmans, et cette pratique est peu familière à la plupart des gens qui se rattachent à l'hindouisme. Nous pourrions donc nous attendre à ce que le passage de l'incinération à l'inhumation ait été perçu comme un éloignement radical par rapport aux pratiques traditionnelles et que cette nouvelle pratique n'ait pas été acceptée d'emblée.

Avant de parler des stratégies déployées par les familles des Maaveerar et par les civils tamouls pour promouvoir l'acceptation de cette nouvelle pratique funéraire, voyons quelles ont été les justifications officielles avancées par les chefs des TLTE. Interrogé sur les raisons du changement de rituel, M. Pontyagam, responsable des affaires se rapportant aux Maaveerar, a déclaré:

Avant 1991, nous les incinérions [les combattants] selon les rituels hindous. Si les parents demandaient les cendres, nous les leur donnions. Mais les chrétiens et les musulmans ne demandaient pas les cendres. Nous avions aussi un problème concernant les soldats chrétiens : les parents ne voulaient pas les incinérer. Nos leaders se sont rencontrés pour étudier ce que d'autres pays comme les États-Unis et l'Angleterre faisaient pour leurs soldats. Ils ont constaté que les Anglais et les Américains enterraient leurs soldats. Ils ont donc décidé de procéder de la même façon ${ }^{4}$.

Ensuite, interrogé sur la réaction des familles hindoues, Pontyagam a répondu de la façon suivante:

Oui, les familles hindoues ont approuvé parce qu'ils [les leaders des TLTE] leur ont expliqué qu'il s'agissait d'une coutume internationale. Avant ça, il y avait des problèmes. Alors, ils ont décidé, plutôt, Prabhakaran ${ }^{5}$ a décidé ce qu'il fallait faire.
En jetant un coup d'œil sur des photos des Tuillum Illam, nous pouvons reconnaître dans leur structure le modèle des cimetières de guerre occidentaux; les Tuillum Illam ressemblant plus aux cimetières de guerre occidentaux qu'aux autres cimetières de la région.

Il n'est pas surprenant que les TLTE aient choisi d'adopter les pratiques funéraires des armées occidentales. C'est que les Tigres n'aiment pas l'épithète de «terroristes». Ils revendiquent plutôt le statut de combattants pour la libération. Ce faisant, ils ne ratent jamais une occasion de souligner qu'ils constituent une armée régulière, faisant remarquer, par exemple, qu'ils portent des uniformes. L'acceptation des pratiques funéraires des militaires occidentaux peut donc être considérée comme la conséquence logique de cette revendication des Tigres.

Ce qui est surprenant, c'est de constater que l'explication officielle de la transition de la crémation à l'inhumation n'est jamais mentionnée par les civils ou par les combattants. Ces deux groupes ont plutôt tendance à donner d'autres explications pour le changement. Lorsque nous étions sur le terrain, nous avons interviewé des combattants des TLTE, des civils tamouls, résidant dans les territoires contrôlés par les TLTE ou dans des territoires contrôlés par le gouvernement, ainsi que des Tamouls vivant maintenant en Italie. Les propos recueillis donnent des explications différentes à propos du passage de l'incinération à l'inhumation, mais aucun ne fait référence à l'explication officielle. Sans doute cette explication n'est-elle pas significative ni acceptable pour les civils tamouls et pour les familles des défunts. En effet, lorsqu'une fille ou un fils, une sœur ou un frère est inhumé plutôt qu'incinéré selon la tradition, il est probable que la famille cherche des explications plus significatives que celle faisant référence à la tradition militaire occidentale.

Il existe deux justifications principales ${ }^{6}$ pour expliquer le changement des pratiques funéraires des Tigres tamouls. La première met en évidence le besoin de mémoire, la seconde inscrit l'inhumation dans le courant de la tradition hindoue.

\section{LES TUILLUM ILLAM EN TANT QUE LIEUX DE LA MÉMOIRE}

Pour expliquer le processus par lequel les Tuillum Illam en viennent à être considérés comme des lieux de mémoire, nous citons quelques extraits des propos recueillis au Sri Lanka au cours de notre travail de terrain. Par exemple, un combattant de Vavuniya a déclaré: "Il s'agit d'un lieu de commémoration, si vous les brûlez [les Maaveerar], alors le passé sera détruit.» De la même façon, un responsable du Tuillum Illam de Koppai nous a dit:

$\mathrm{Au}$ début, nous faisions des incinérations [les Maaveerar]. Puis, nous nous sommes dit: "Ce n'est pas bien, ce serait mieux d'avoir un lieu pour honorer leur mémoire. » Si nous avions un monument, nous pourrions faire une célébration à chaque année, leur famille pourrait venir les visiter, ce qu'elles font régulièrement.

À Jaffna, un civil a affirmé :

Dans ce contexte, nous avions besoin d'un endroit pour rendre notre peuple heureux. Quand nos enfants nous posent la question [en indiquant le Tuillum Illam]: "Qu'est-ce que c'est?», nous répondons "C'est un endroit dédié à ceux qui ont sacrifié leur vie pour la liberté du Tamil Eelam.»

Un garçon tamoul qui vit en Italie a fait remarquer que:

Les Maaveerar sont des gens qui protègent la terre, notre patrie. Si nous les incinérons, ils deviennent cendres, et ils disparaîtront. Pour que leur souvenir reste vivant, les Tigres les enterrent et construisent des tombeaux. Ils écrivent sur leur pierre tombale: "Cet individu est mort pour défendre sa patrie» et, de cette façon, ils [les Maaveerar] restent longtemps parmi nous.

Enfin, la sœur d'un combattant mort à l'Elephant Pass a précisé: "Nous devons conserver leurs corps [ceux des Maaveerar], du moins, leurs os doivent être conservés. » Nous pourrions discuter ici la nécessité d'avoir des tombeaux pour avoir des lieux de la mémoire. Ce serait alors oublier que dans l'hindouisme, comme le disent Maurice Bloch et Jonathan Parry:

Rien des individus n'est préservé, pour qu'il n'y ait pas de continuité, même symbolique, entre le groupe et le défunt. Tout ce qui reste du défunt est oblitéré: le corps est d'abord incinéré, les cendres sont ensuite dispersées dans le Gange qui, lui, se déverse dans l'océan. L'objectif ultime paraît donc être la dissolution complète du cadavre (1982, p. 36).

Or, une telle dissolution du corps implique l'absence d'un espace public pour commémorer les défunts. Même s'il existe des rituels postcrématoires, au cours desquels sont vénérés les ancêtres (Knipe, 1977), ces rituels sont accomplis seulement par les membres de la famille dans l'espace privé de la maison. L'arrière-plan culturel explique donc ce manque de corrélation entre l'incinération et la présence d'un lieu public dédié à la mémoire des défunts. 


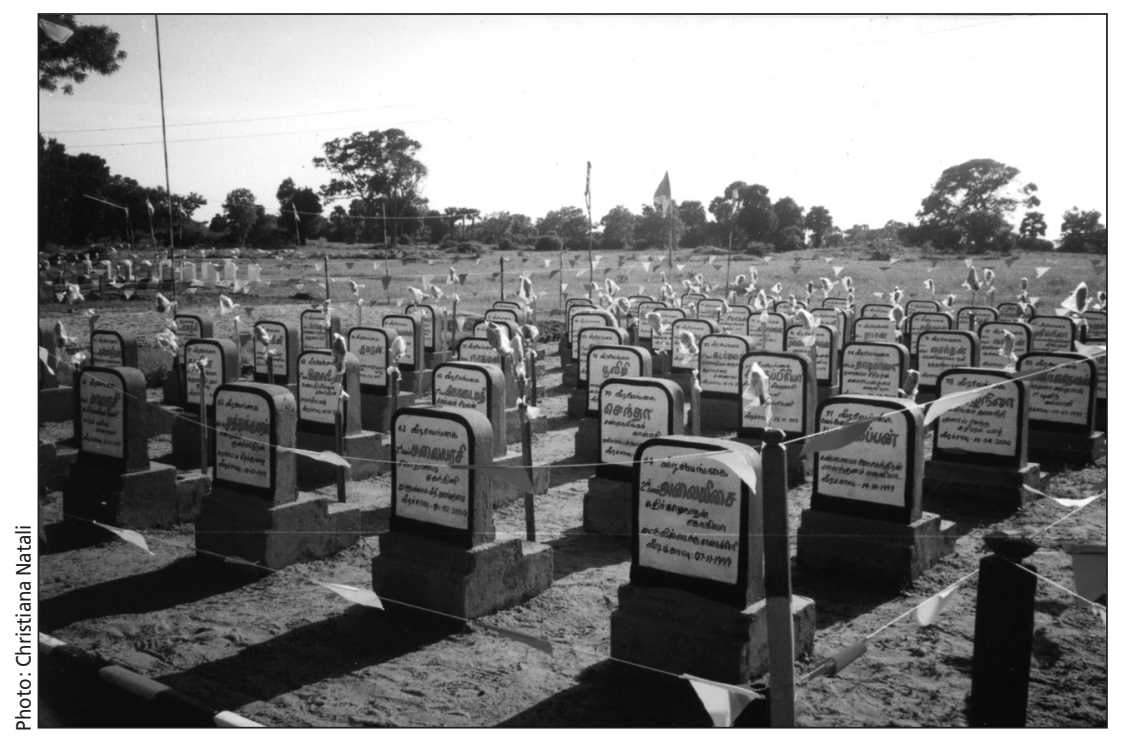

Cimetière d'Uduthurai,

section des cénotaphes.

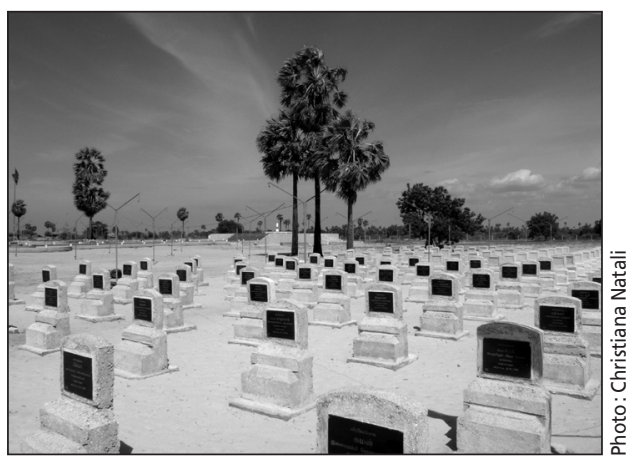

- Tuillam Illam de Tunukkai, section des cénotaphes.

Tuillam Illam de Koppai (Jaffna), section des tombes.
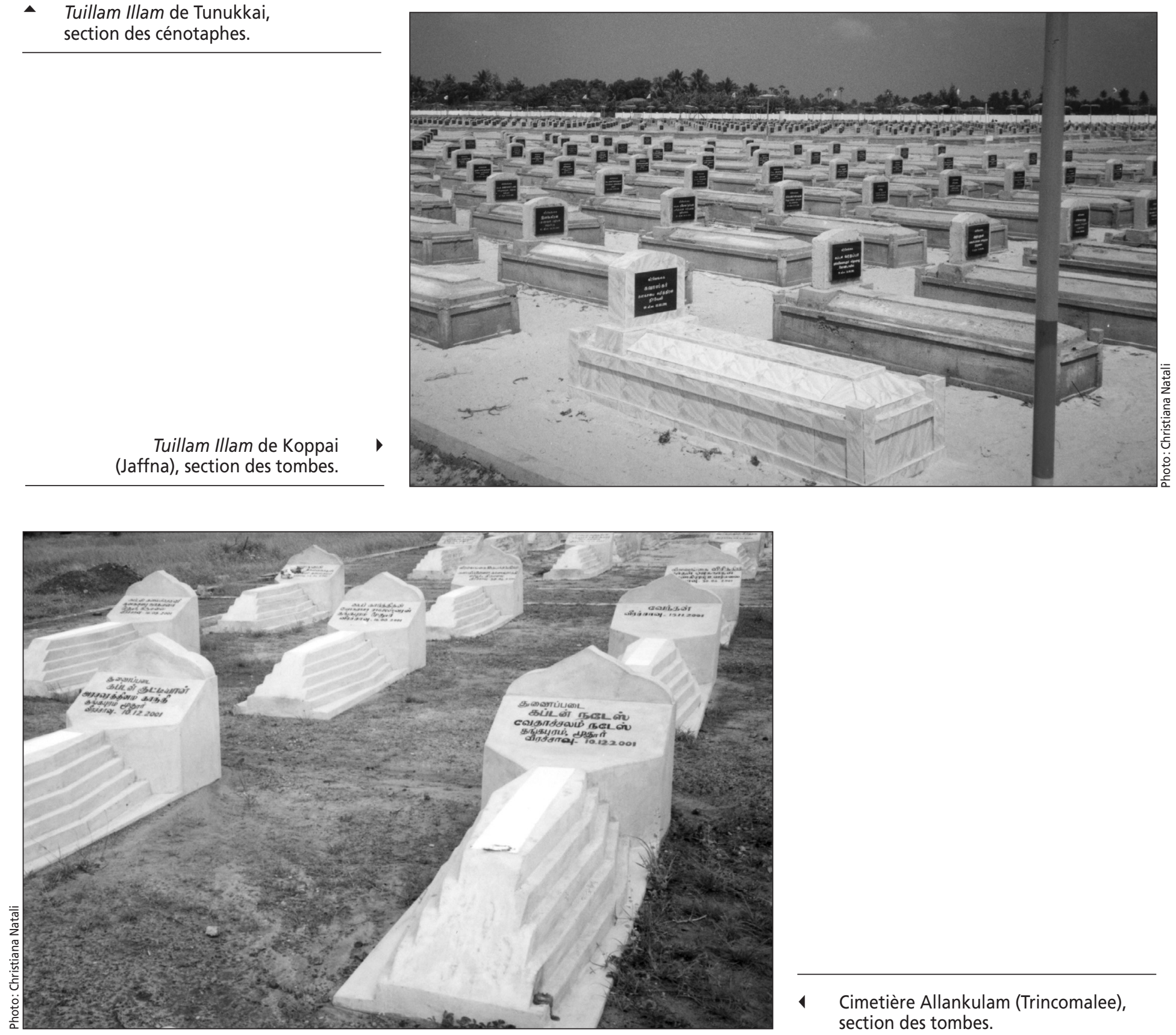

1 Cimetière Allankulam (Trincomalee), section des tombes. 
D'après les témoignages recueillis, la perception des Tuillum Illam comme lieux de mémoire expliquerait leur destruction par l'armée du Sri Lanka7. M. R. Narayan Swamy, un journaliste, décrit la conquête de Jaffna de la façon suivante:

Un jour ou l'autre, il est évident que Prabhakaran va reconquérir Jaffna.

Peu lui importe le coût de l'opération, il n'y a rien d'autre qui puisse venger l'humiliation de 1995, quand les troupes sri-lankaises victorieuses défilèrent dans cette ancienne ville tamoule pendant les célébrations nationales. L'armée du Sri Lanka a profané les pierres tombales des martyrs tamouls, et cette profanation n'a pas été oubliée par les Tigres tamouls. (2002, p. 355 ; c'est nous qui soulignons.)

À l'entrée des Tuillum Illam de Koppai et de Naundil, les visiteurs peuvent voir les restes rassemblés des tombes et des cénotaphes dévastés. Accompagnant ces pièces, un livre de pierre avec l'inscription suivante:

Après notre déportation en 1995, l'armée du Sri Lanka a endommagé et détruit les monuments de nos héros de guerre, trésors de notre peuple.

Nous avons conservé les pierres qui ont échappé à ce désastre.

Inclinons nos têtes et recueillons-nous un moment pour rendre hommage à leur mémoire.

La destruction des tombes par l'armée sri-lankaise peut être considérée comme une preuve de l'importance accordée aux Tuillum Illam par les soldats gouvernementaux dans leur lutte contre les Tigres. Pour mieux comprendre l'importance symbolique des terrains de sépulture des TLTE, nous devons nous concentrer sur les analogies fonctionnelles existant entre ces lieux et les cimetières militaires de la tradition occidentale.

\section{ANALOGIES FONCTIONNELLES AVEC LES CIMETIĚRES DE GUERRE DANS LA TRADITION MILITAIRE OCCIDENTALE}

Nous avançons que les Tuillum Illam partagent plusieurs fonctions avec les cimetières de la tradition occidentale. Comme l'observe George Mosse (1990), le fait de rassembler les restes de tous les soldats morts au même endroit est un moyen de souligner l'importance de leur geste pour la nation et, par conséquent, de concentrer l'attention de la population sur leur sacrifice. C'est exactement dans cette perspective que nous pourrions lire les mots de Prabhakaran qui, en parlant des cimetières, affirme que «Les sépultures des héros Tigres morts au combat seront les fondations de notre nouvelle nation » (paru dans le journal des TLTE en 1995).
Une autre fonction importante des cimetières de guerre en Occident est leur utilisation comme lieux commémoratifs. Cette utilisation est aussi celle des Tuillum Illam. Les Maaveerar sont commémorés le 27 novembre, date considérée comme le jour de la mort du premier Tigre. Au cours de cette journée, les TLTE honorent la mémoire de leurs combattants morts dans le monde entier. Les Tamouls de la diaspora organisent des célébrations publiques dans les théâtres ou dans les salles communautaires $^{8}$, alors qu'au Sri Lanka les cérémonies ont lieu dans les Tuillum Illam mêmes. Ces cérémonies commencent l'après-midi, alors que des gens se rendent à un Tuillum Illam, pour placer des fleurs, de l'encens, du camphre et des bougies près des tombeaux. En signe de deuil, les femmes pleurent et crient. Le jour des Maaveerar est un événement politique significatif, non seulement à cause de l'implication très étendue des civils, mais aussi parce qu'il y a diffusion du discours annuel de Prabhakaran à travers les haut-parleurs placés dans tous les Tuillum Illam. Cheran le souligne, le discours de Prabhakaran est considéré comme «une sorte de discours du Trône, dans lequel il traite des victoires militaires et de la situation sur le territoire en général. Il y expose aussi différents plans d'avenir ainsi qu'il analyse la situation politique du moment»(2001, p. 17). En ce sens, les Tuillum Illam sont le lieu privilégié de la mise en place d'une "rhétorique intentionnelle », un élément central au sein du processus de construction de la nation, comme le rappelle Elizabeth Tonkin. Cette dernière remarque que la «rhétorique intentionnelle» est employée "pour convaincre le peuple de l'existence d'une identité sociale qui n'existe que par les Tuillum Illam» (1992, p. 130).

Pour conclure l'analyse des analogies fonctionnelles, nous pouvons mentionner que même dans les cimetières de guerre appartenant à la tradition occidentale, certains symboles peuvent donner lieu à différentes interprétations, comme l'explique George Mosse :

Les cimetières anglais sont construits autour de la Croix du Sacrifice et de la Pierre du Souvenir. Pour reprendre les mots de Rudyard Kipling, la Croix du Sacrifice se présente comme « $a$ stark sword brooding in the bossom of the cross ». Le symbolisme de la Croix [...] reste quand même vague. Il pourrait signifier le sacrifice d'une vie à la guerre ou encore l'espoir de la résurrection. [...] La Pierre du Souvenir et la Croix du Sacrifice ont été associés au symbolisme chrétien d'influence dominante dans le cimetière mais à l'origine, pour l'architecte Sir Edwin
Lutyens, la Pierre constituait un symbole appartenant à un panthéon non-chrétien. [...] Par la suite, la Pierre du Souvenir a parfois été désignée sous le nom de «l'autel», ce qui lui a conféré une signification religieuse comme c'était déjà le cas pour la Croix du Sacrifice (1990, p. 85).

De la même façon, dans le Tuillam Illam, la «flamme du sacrifice» qui brûle sur le plateau central pourrait être comparée, comme le suggère le recteur de l'Université de Jaffna, à la flamme de l'Arc de triomphe de l'Étoile à Paris, en même temps qu'elle pourrait être perçue comme un remplaçant symbolique du feu crématoire.

\section{LES TUILLUM ILLAM \\ EN TANT QUE TEMPLES}

Nous allons maintenant examiner une deuxième tendance dans le discours populaire pour justifier le changement des pratiques funéraires. Selon cette interprétation, qui vient surtout des civils, l'inhumation s'inscrit dans le courant principal de la tradition hindoue. Comme nous l'avons vu précédemment, dans l'hindouisme, il y a des exceptions à l'incinération des corps. Dans le contexte de la guerre civile au Sri Lanka, ces pratiques funéraires exceptionnelles sont mises en rapport avec les pratiques funéraires réservées aux combattants des TLTE pour donner une impression de continuité culturelle. L'analyse des exceptions à l'incinération chez les hindouistes va clairement au-delà du propos de notre article. C'est pourquoi nous nous bornerons à souligner que ces exceptions sont justifiées pas des raisons financières (les pauvres n'ont pas les revenus nécessaires pour incinérer leurs morts) et religieuses. Du point de vue religieux, le rituel crématoire en tant que sacrifice offert aux dieux comporte maintes implications: par exemple, en cas de "mauvaise mort», d'un décès inattendu et prématuré (comme la mort provoquée par une noyade, par un acte violent ou par certaines infirmités), le corps ne constitue pas un sacrifice approprié aux divinités et en conséquence, il n'est pas incinéré ${ }^{9}$.

Il y a d'autres circonstances où le corps est inhumé, par exemple lorsque le mort est un enfant ou un ascète. En ce qui concerne les enfants, il y a des traces interculturelles de différentes pratiques pour ceux qui meurent dans les premières années de leur existence. Les raisons qui expliquent ce traitement spécial réservé aux enfants dans l'hindouisme ont donné lieu à un débat passionné (voir Das, 1976, et Malamoud, 1982). Les critiques ont proposé maintes interprétations, et quelques-unes insistent sur la position liminale de l'enfant à l'intérieur de la société. Pour ce qui est des 
ascètes, ils peuvent eux aussi être considérés comme des figures liminales, et ce, par leur exclusion du système de castes et par leur statut symbolique, sur le seuil (limen), entre la vie et la mort. En effet, l'inhumation des ascètes est justifiée par leur renoncement à la vie ordinaire, comme l'explique Charles Malamoud (1989, p. 65):

La cérémonie complexe qui marque l'entrée en "renoncement» consiste à laisser s'éteindre les feux sacrificiels après y avoir fait brûler, ultime oblation, ultime combustible, les divers ustensiles du sacrifice. Les feux ne sont pas abolis pour autant: ils sont intériorisés, inhalés, on les fait «remonter» en soi [...] cuit de l'intérieur, et de son vivant même, le samnyasin [ascète]

n'a pas à être cuit après sa mort : il n'est donc pas incinéré, mais inhumé [...] En intériorisant leurs feux, ils ont aussi aboli la possibilité d'être transportés vers une divinité qui leur soit extérieure. En s'instituant d'em-

blée comme offrande, et en persistant jusqu'au bout dans ce rôle, ils ont fait de leur propre personne, de leur atman identifié au Soi universel, leur divinité.

Grâce à cette exception qu'est l'inhumation des ascètes, les civils tamouls ont la possibilité de placer la pratique de l'inhumation dans la tradition hindouiste. Pour comprendre l'analogie symbolique entre l'inhumation des ascètes et celle des Maaveerar, nous devons examiner comment les combattants des TLTE se présentent eux-mêmes: ils se présentent comme des hommes et des femmes qui refusent les «mauvaises coutumes» des gens ordinaires: ils ne boivent pas, ils ne fument pas et ils n'ont pas de relations sexuelles interdites ${ }^{10}$. L'abstention d'alcool et de cigarettes concerne surtout les hommes, puisque dans la culture tamoule les femmes ne sont pas censées boire ou fumer. À propos des femmes combattantes, leur caractère particulier réside dans leur pureté, comme le confirme Radhika Coomaraswamy:

Dans l'idéal du TLTE, les groupes de guérilla féminins doivent projeter une image de pureté et de virginité. [...] Les femmes sont décrites comme étant pures et vertueuses. Leur chasteté, leur contribution à un but commun ainsi que le sacrifice de leur vie sociale seraient les sources de leur force. Se doter d'une armée de femmes vierges fait en sorte que la notion de pureté, fondée sur un déni, devient un élément de la construction sociale de l'identité féminine selon la vision du monde du TLTE (Coomaraswamy, d'après Schrijvers, 1999, p. 316).

IL EXISTE DEUX JUSTIFICATIONS PRINCIPALES POUR EXPLIQUER LE CHANGEMENT DES PRATIQUES FUNÉRAIRES DES TIGRES TAMOULS: LA PREMIÈRE MET EN ÉVIDENCE LE BESOIN DE MÉMOIRE, LA SECONDE INSCRIT L'INHUMATION DANS LE COURANT

DE LA TRADITION HINDOUE.

Michael Roberts suggère que le modèle ascétique des combattants implique «l'influence de la tradition hindouiste du tapas (la force par l'abstinence) ainsi que celle de l'auto-discipline des révolutionnaires maoïstes» (1996, p. 256). L'attitude ascétique des combattants est aussi un des sujets faisant partie de la production cinématographique des TLTE. À ce propos, Peter Schalk, en rapportant l'intrigue d'un film sur les Tigres Noirs - les commandos suicides-, a observé que :

Le héros du film est présenté comme un tavan, un ascète, en raison de son comportement. Même s'il est en âge de se marier, il ne semble pas avoir de compagne [...] Vivant dans le groupe des Tigres Noirs, il semble se consacrer entièrement à une cause sacrée [c'est-à-dire, libérer la patrie tamoule] (1997, p. 160).

L'association symbolique entre combattants et ascètes ne se borne pas seulement à leur conduite. Après leur mort, les combattants, aussi bien que les ascètes, sont vénérés et perçus comme des dieux. Quand nous avons demandé si les Tuillum Illam étaient des cimetières ou non, tous les gens interviewés ont répondu: «Comment pouvez-vous demander une telle chose? Les Tuillam Illam sont des temples où sont plantés [enterrés] les dieux.» Prenant en considération les conduites imposées aux visiteurs des Tuillum Illam, les pratiques établies autour des Tuillum Illam sont totalement contraires à celles observées lors de la visite de cimetières. L'absence des femmes et l'obligation de prendre un bain lorsque l'on sort des lieux de sépulture sont les différences les plus évidentes.

Lidentification des Maaveerar avec les dieux, qui exigerait certainement une analyse plus détaillée, n'est pas refusée par les TLTE, et ce, en dépit de leur nature séculière. Au cours de notre travail de terrain, nous avons observé que non seulement les civils, mais aussi les non-combattants ayant des contacts avec le mouvement sont persuadés que les Maaveerar sont des dieux et que les Tuillum Illam sont des temples. À notre avis, les TLTE ne refusent pas cette interprétation, car elle est nécessaire pour faire en sorte que les nouveaux rituels funéraires soient acceptés. En effet, comme le dit Paul Connerton dans son ouvrage How Societies Remember:

Toute forme de commencement contient un élément de mémoire, particulièrement lorsqu'un groupe social se mobilise pour se donner un tout nouveau départ. Une telle tentative de re-commencement, par sa nature même, laisse une grande place à l'arbitraire. [...] Mais l'idée de nouveauté absolue est inconcevable. Non seulement est-il très difficile de se donner un tout nouveau départ, mais il y a trop d'anciennes loyautés et d'anciennes habitudes qui empêchent le nouveau projet de prendre la place de celui qui précédait et qui était déjà bien établi (1989, p. 6).

En conséquence, ce n'est pas surprenant de voir, comme l'observe Chandrakanthan, que même Prabhakaran compare les combattants aux ascètes:

Prabhakaran, le leader du TLTE, exige du peuple qu'il vénère ceux qui sont morts à la bataille de Eelam comme s'il s'agissait de sannyasis (d'ascètes) ayant renoncé à leurs désirs personnels et ayant réussi à transcender l'égoïsme de leur existence pour se consacrer à une cause commune de grande vertu (2000, p. 164).

En conclusion, mentionnons que l'attitude des TLTE, en ce qui concerne l'identification des Maaveerar aux dieux, apparaît ambiguë mais nécessaire. D'une part, les Tuillum Illam sont des lieux où les valeurs de la future nation sont mises en place: le refus idéologique de toute différence parmi les gens (différences de caste, de milieu et de genre) est symboliquement représentée par l'uniformité des rituels funéraires et de la construction des tombes. D'autre part, cette idée que les Tuillum Illam soient des temples où les Maaveerar/dieux sont vénérés permet aux TLTE d'éviter la rupture avec les sentiments religieux des civils, tout en assurant le consentement populaire au nouveau projet de création de la nation. 


\section{Bibliographie}

BLOCH, M. et J. PARRY (dir.) (1982). Death and the Regeneration of Life, Cambridge, Cambridge University Press.

CHANDRAKANTHAN, A. J. V. (2000). «Eelam Tamil Nationalism. An Inside View », dans J.A. Wilson, Sri Lankan Tamil Nationalism. Its Origins and Development in the 19th and 20th Centuries, London, Hurst, p. $157-175$

CHERAN, R. (2001). "The Sixth Genre: Memory, History and the Tamil Diaspora Imagination ", Colombo, Marga Monograph Series on Ethnic Reconciliation, VII.

CONNERTON, P. (1989). How Societies Remember, Cambridge, Cambridge University Press.

DAS, V. (1976). "The Uses of Liminality: Society and Cosmos in Hinduism ", Contributions to Indian Sociology, vol. X, $\mathrm{n}^{\circ} 2$, p. 245-263.

FUGLERUD, I. et O. FUGLERUD (1999). Life on the Outside. The Tamil Diaspora and Long Distance Nationalism, London, Pluto Press.

FUGLERUD, O. (2001). « Time and Space in the Sri-Lanka-Tamil Diaspora », Nations and Nationalism, vol. 7, $n^{\circ}$ 2, p. 195-213.

KNIPE, D.M. (1977). «Sapindiikarana: The Hindu Rite of Entry into Heaven ", in F.E. Reynolds et E.H. Waugh (dir.), Religious Encounters with Death, University Park and London, Pennsylvania State University Press, p. 111-124.

MALAMOUD, Ch. (1989). Cuire le monde. Rite et pensée dans l'Inde ancienne, Paris, La Découverte.

MOSSE, G.L. (1990), Fallen Soldiers: Reshaping the Memory of the World Wars, New York, Oxford University Press.

NATALI, C. (2002). «Uno spazio scenico per la memoria: la commemorazione dei caduti tamil attraverso la danza», dans A. Destro (dir.), Antropologia dello spazio. Luoghi e riti dei vivi e dei morti, Bologna, Patron, p. 77-111.

NATALI, C. (2004), Sabbia sugli dèi. Pratiche commemorative tra le Tigri tamil (Sri Lanka), Torino, il Segnalibro

ROBERTS, M. (1996). «Filial Devotion in Tamil Culture and the Tiger Cult of Martyrdom ", Contributions to Indian Sociology, vol. $30, n^{\circ} 2$, p. $245-272$.

SCHALK, P. (1997). «The Revival of Martyr Cults among Ilavar», Temenos, $\mathrm{n}^{\circ} 33$, p. 151-190.

SCHRIJVERS, J. (1999). «Fighters, Victims and Survivors: Constructions of Ethnicity, Gender and Refugeeness among Tamils in Sri Lanka", Journal of Refugees Studies, vol. XII, no 3 .

SWAMI, N. (2002). Tigers of Lanka. From Boys to Guerrillas, Colombo, Vijitha Yapa.

TONKIN, E. (1992). Narrating Our Pasts. The Social Construction of Oral History, Cambridge, Cambridge University Press.

\section{Notes}

1. Ce texte est la traduction française de la communication présentée à Leeds le 31 mars 2005 à l'occasion de la conférence annuelle de la BASAS (British Association for South Asian Studies). Les traductions en français sont de Frontières.

2. Tamil Eelam fait référence à l'État séparé revendiqué par les TLTE (Tigres de la Libération du Tamil Eelam).

3. Quelquefois les chrétiens sont enterrés près de l'endroit où les hindous pratiquent l'incinération.

4. Communication personnelle (le 7 décembre 2002).

5. Nous observons que Prabhakaran est censé prendre toutes les décisions capitales qui concernent les combattants. Les membres des TLTE eux-mêmes nous ont expliqué que, même si beaucoup de décisions sont prises d'un commun accord, il vaut mieux les attribuer au chef. De cette façon, les gens les accepteront plus facilement.

6. Même si la description détaillée de toutes ces interprétations va au-delà de notre projet d'étude, il est nécessaire de mentionner que, pour certains combattants (mais non pas pour les civils), l'inhumation des morts est considérée comme un retour aux pratiques des anciens Tamouls, qui enterraient en effet leurs guerriers morts au champ d'honneur. Des références aux usages de l'époque de Chankam sont présentes aussi à l'intérieur de quelques publications des TLTE. Comme l'explique Fuglerud, «The ideological project of the TLTE is directed towards homologising the pre- and post-colonial situation, of linking the present claim for statehood with the restoration of authentic Tamil culture » (2001, p. 203). Pour en savoir plus, voir Cheran (2001) et Natali (2004).

7. Nous devons ici rappeler que la destruction des Tuillum Illam n'est officiellement reconnue ni par le gouvernement sri-lankais, ni par les organisations internationales, par exemple le Comité international de la Croix-Rouge (CICR).

8. Pour des détails supplémentaires à propos des cérémonies hors du Sri Lanka, voir Cheran (2001) pour ce qui est de la situation au Canada, et Natali (2002) pour l'Italie.

9. Cela n'implique pas nécessairement l'inhumation du corps: parfois, le corps est placé dans le fleuve où il s'en va à la dérive (même les corps des enfants subissent souvent ce traitement).

10. Rapports sexuels avant le mariage ou hors mariage. 